\title{
A CONSTRUCTIVE CHARACTERIZATION OF VERTEX COVER ROMAN TREES
}

\author{
Abel Cabrera Martínez \\ Universitat Rovira $i$ Virgili \\ Departament d'Enginyeria Informàtica i Matemàtiques \\ Av. Països Catalans 26, 43007 Tarragona, Spain \\ e-mail: abel.cabrera@urv.cat \\ Dorota KuZIAK \\ Universidad de Cádiz \\ Departamento de Estadística e Investigación Operativa \\ Escuela Politécnica Superior de Algeciras \\ Av. Ramón Puyol s/n, 11202 Algeciras, Spain \\ e-mail: dorota.kuziak@uca.es \\ AND \\ ISMAel G. Yero \\ Universidad de Cádiz \\ Departamento de Matemáticas \\ Escuela Politécnica Superior de Algeciras \\ Av. Ramón Puyol s/n, 11202 Algeciras, Spain \\ e-mail: ismael.gonzalez@uca.es
}

\begin{abstract}
A Roman dominating function on a graph $G=(V(G), E(G))$ is a function $f: V(G) \rightarrow\{0,1,2\}$ satisfying the condition that every vertex $u$ for which $f(u)=0$ is adjacent to at least one vertex $v$ for which $f(v)=2$. The Roman dominating function $f$ is an outer-independent Roman dominating function on $G$ if the set of vertices labeled with zero under $f$ is an independent set. The outer-independent Roman domination number $\gamma_{o i R}(G)$ is the minimum weight $w(f)=\sum_{v \in V(G)} f(v)$ of any outer-independent Roman dominating function $f$ of $G$. A vertex cover of a graph $G$ is a set of vertices that covers all the edges of $G$. The minimum cardinality of a vertex cover is denoted by $\alpha(G)$. A graph $G$ is a vertex cover Roman graph
\end{abstract}


if $\gamma_{o i R}(G)=2 \alpha(G)$. A constructive characterization of the vertex cover Roman trees is given in this article.

Keywords: Roman domination, outer-independent Roman domination, vertex cover, vertex independence, trees.

2010 Mathematics Subject Classification: 05C69, 05C05, 05 C75.

\section{REFERENCES}

[1] H. Abdollahzadeh Ahangar, M. Chellali and V. Samodivkin, Outer independent Roman dominating functions in graphs, Int. J. Comput. Math. 94 (2017) 25472557. doi:10.1080/00207160.2017.1301437

[2] E.J. Cockayne, P.A. Dreyer, S.M. Hedetniemi and S.T. Hedetniemi, Roman domination in graphs, Discrete Math. 278 (2004) 11-22. doi:10.1016/j.disc.2003.06.004

[3] T. Gallai, Über extreme Punkt-und Kantenmengen, Ann. Univ. Sci. Budapest. Eötvös Sect. Math. 2 (1959) 133-138.

[4] T.W. Haynes, S.T. Hedetniemi and P.J. Slater, Fundamentals of Domination in Graphs (Marcel Dekker, New York, 1998).

[5] T.W. Haynes, S.T. Hedetniemi and P.J. Slater, Domination in Graphs: Advanced Topics (Marcel Dekker, New York, 1998).

[6] M.A. Henning, A characterization of Roman trees, Discuss. Math. Graph Theory 22 (2002) 325-334. doi:10.7151/dmgt.1178

[7] M.A. Henning and W.F. Klostermeyer, Italian domination in trees, Discrete Appl. Math. 217 (2017) 557-564. doi:10.1016/j.dam.2016.09.035

[8] B. Randerath and L. Volkmann, Characterization of graphs with equal domination and covering number, Discrete Math. 191 (1998) 159-169. doi:10.1016/S0012-365X(98)00103-4

[9] I. Stewart, Defend the Roman Empire!, Sci. Amer. 281 (1999) 136-138. doi:10.1038/scientificamerican1299-136

Received 22 June 2017

Revised 5 October 2018

Accepted 5 October 2018 